\title{
Hormonal Correlates of Hand Preference in Free-ranging Primates
}

\author{
G.C. Westergaard, Ph.D., T.J. Chavanne, M.A., I.D. Lussier, B.S., S.J. Suomi, Ph.D., \\ and J.D. Higley, Ph.D.
}

In this research we examined hormonal correlates of hand preference in free-ranging primates. Specifically, we tested the hypothesis that levels of the stress hormone cortisol and the male sex hormone testosterone are correlated with handedness in male rhesus macaques (Macaca mulatta). We found significant positive relationships between cortisol and testosterone levels sampled during adolescence and the frequency of right-versus left-hand use sampled during adulthood. These data indicate that adolescent measures of cortisol and testosterone are correlated with hemispheric specialization in adult free-ranging primates.

[Neuropsychopharmacology 23:502-507, 2000]

Published by Elsevier Science Inc.
KEY WORDS: Cortisol; Testosterone; Laterality; Development; Rhesus macaques

Approximately $90 \%$ of the human population is righthanded and the corresponding left-hemisphere specialization for manual control is thought to have contributed to the evolution of several species-typical characteristics including language and other higher-order cognitive processes. Because of the presumed importance of hemispheric specialization, a great deal of attention has been paid to understanding proximate factors associated with lateral asymmetries and the reduced incidence of left-handedness. In recent years considerable evidence has emerged suggesting a relationship between stress reactivity and lateral asymmetries. For example, research with young rhesus monkeys indicates

From the Division of Research and Development (GCW, TJC IDL), LABS of Virginia Inc., Yemassee, SC; Laboratory of Comparative Ethology (SJS), National Institute of Child Health and Human Development, Poolesville, MD, and Laboratory of Clinical Studies (JDH), National Institute on Alcohol Abuse and Alcoholism, Poolesville, MD.

Address correspondence to: G.C. Westergaard, Ph.D., Division of Research, LABS of Virginia, Inc., 95 Castle Hall Road, P.O. Box 557, Yemassee, SC 29902. Tel.: 843-589-5190, Ext. 23; Fax: 843-589-5290; E-mail: Gwprimate@aol.com

Received November 9, 1999; revised April 25, 2000; accepted May 4, 2000 . that high levels of circulating cortisol are associated with fearful behavior and high right-frontal activity (Kalin et al. 1998). This finding is consistent with results from human studies showing a preferential role for the right-hemisphere in emotion-induced cortisol release (Wittling and Pfluger 1990; also see Henry 1993) and further supports the view that neonatal stress and its physiological consequences influence the emergence of hemispheric specialization.

The steroid hormone testosterone has been linked to left-handedness in humans (Geschwind and Galaburda 1985a, 1985b; Witelson 1991), generating considerable controversy about processes that underlie this relationship. One perspective holds that high levels of testosterone in utero are associated with left-handedness, ambidexterity, and reduced cerebral dominance. In this view high testosterone inhibits cortical development in certain regions of the left hemisphere while promoting development in corresponding regions in the right hemisphere (Geschwind and Galaburda 1985a, 1985b). A competing perspective is that low prenatal levels of testosterone contribute to the development of left-handedness and reduced functional asymmetry by reducing the probability of cell death and axon elimination in the temporo-parietal region (Witelson 1991). Witelson based this hypothesis on handedness-related differences in the corpus callo- 
sum and reports of a disproportionately high incidence of non-right-handedness among homosexuals (Lindesay 1987; McCormick et al. 1990). More recent empirical research with human adults has shown that left-handers have lower salivary testosterone than do their righthanded counterparts (Moffat and Hampson 1996), demonstrating a systematic handedness-related difference in bioactive testosterone concentrations consistent with Witelson's (1991) view.

Lateralized behavior and corresponding morphological asymmetries have been noted in numerous primate species, indicating that primate laterality is phylogenetically widespread (Bradshaw and Rogers 1993; MacNeilage et al. 1987; also see Warren 1980). Several studies in this area have focused on the behavior of the rhesus monkey (Macaca mulatta), a primate species widely used to model human psychobiological processes (Schneider and Suomi 1992; Westergaard et al. 1997, 1998). Rhesus monkeys share approximately 90 $94 \%$ of their genetic material with humans and it is likely that this high level of genetic similarity largely accounts for compelling parallels between the two species in terms of morphology, physiology, and expression of complex behavior (Ciochon and Fleagle 1985; Fleagle 1986). Group-level age-based differences in the direction of rhesus handedness have been found in laboratory research showing left-hand bias in young-adult animals and right-hand bias in geriatric animals (Westergaard and Lussier 1999). Research with nursery-reared rhesus monkeys has shown that left-hand bias emerges prior to one year of age (Westergaard et al. 1997) and that plasma cortisol levels are positively associated with the frequency of right- versus left-hand use during the latter half of the first postnatal year (Westergaard, unpublished observation). In addition, Drea et al. (1995) have found greater strength of hand preference (independent of direction) in rhesus monkeys with high levels of neonatal testosterone, suggesting a broad influence of the CNS-pituitary-testicular axis on brain asymmetries and providing evidence consistent with testosterone-influenced brain differentiation.

In the present research we examined relationships among cortisol, testosterone, and handedness in freeranging rhesus macaques. We tested the hypothesis that differences in hormone functioning are correlated with hemispheric specialization and manifested by a positive correlation between cortisol and testosterone levels and the frequency of right- versus left-hand use. We sampled cortisol and testosterone from adolescents aged between 1 and 5 years, and we subsequently recorded hand preferences from these same animals as adults aged between 8 and 12 years. To the best of our knowledge this is the first study to examine hormonal correlates of handedness in free-ranging primates. The research described in this report evaluated the relationship among cortisol, testosterone, and handedness in nonhuman primates in order to extend our knowledge of hemispheric specialization and manual laterality in humans.

\section{METHODS}

\section{Subjects}

The research subjects were 34 male rhesus macaques (Macaca mulatta). The study subjects belong to a colony of 4000 free-ranging monkeys that reside on a 475-acre South Carolina barrier island. At the time of subject selection the population was organized into 35 social groups with an approximate ratio of 2.5 adult females to each adult male. Subjects were selected for physiological sampling when they were juveniles and adolescents, aged 13-59 months (mean age at physiological sampling $=34$ months). These same subjects were selected for hand preference sampling when they were adults, aged 98-147 months (mean age at hand preference sampling $=127$ months).

\section{Physiological Sampling}

We obtained physiological data from subjects that were trapped in baited corrals (diameter of each corral $=10$ $25 \mathrm{~m}$, height of corral walls $=4 \mathrm{~m}$ ). On the first day of a 5-day trapping period, corral feeders were baited with food and then the corral doors were closed. Monkeys voluntarily jumped from the top of the walls down into the corrals to obtain the food, but were unable to escape because they could not jump over the high walls. Following capture, subjects were anesthetized using ketamine hydrochloride $(10 \mathrm{mg} / \mathrm{kg})$ and weighed on a commercial scale accurate to within $0.25 \mathrm{~kg}$. A blood sample was then immediately obtained from each subject via venipuncture and placed on ice. The blood was subsequently centrifuged and the plasma frozen and stored at a temperature of $-70^{\circ} \mathrm{C}$. Samples were assayed for cortisol, testosterone, and adrenocorticotropic hormone (ACTH) by a commercial laboratory using radioimmunoassay. To control for possible confounding influences on hormonal values we recorded the number of minutes elapsed from when the technicians initially entered the corral to when the blood sample was obtained. Time of day was also recorded for each sample. Cortisol samples were available for each of the 34 subjects. Testosterone and ACTH samples were available for 32 subjects. Prior to release each subject was tattooed and marked with dye and fitted with a radio transmitter collar to facilitate field identification and tracking.

\section{Hand Preference Sampling}

We evoked quadrupedal reaching by throwing corn in the vicinity of a focal subject's social group. The subject 
moved to the location to pick up the food. An observer blind to the physiological data and the specific hypotheses of this investigation recorded the hand (left or right) that the subject used to retrieve food. We counted a trial only when a subject maintained both hind limbs and one fore limb on the ground while reaching. To ensure independence between data points, we required subjects to move to the food site before each new reach to ensure postural repositioning. If locomotion did not occur before an animal picked up a food item we did not consider the reach in the tally. We conducted 50 trials per subject over a 6-month period.

To establish inter-observer reliability two observers conducted simultaneous observations at the beginning of the study, the midpoint, and the end of the study. During these sessions both observers concurrently scored the same subject for a 15-min period. We combined these scores in an overall reliability test of the events scored. The reliability of all recorded acts was shown by Cohen's kappa values greater than 0.90 . Both observers were blind to all physiological data and to the specific hypotheses under investigation.

\section{Data Analysis}

We used two measures to characterize hand preference. We first calculated a handedness index $(\mathrm{HI})$ score for each subject using the formula $[(\mathrm{R}-\mathrm{L}) /(\mathrm{R}+\mathrm{L})]$ where $\mathrm{R}=$ the frequency of right-hand responses and $\mathrm{L}=$ the frequency of left-hand responses. This measure quantifies lateral bias along a continuum from strongly lefthanded to strongly right-handed. Positive values indicate a bias toward use of the right hand, and negative values indicate a bias toward use of the left hand. We then used the absolute value of each subject's handedness index score (ABS-HI) to characterize hand preference strength independent of direction. Thus, a subject with an HI score of +.85 would have the same ABS-HI score as would a subject with an HI score of -.85 . This procedure is similar to that which has been used in studies of laterality using human infants as subjects (e.g., Harris and Carlson 1993). We used multiple regression analysis (with age at physiological sampling and age at hand preference sampling held statistically constant) to examine the direction and degree of associations among $\mathrm{HI}$ and ABS-HI scores and levels of plasma cortisol, testosterone, and ACTH. We also conducted linear analysis (Pearson $r$ ) to evaluate relationships between HI and ABS-HI scores and absolute hormone levels. (It must be noted that age at physiological sampling and age at hand preference sampling were not statistically controlled for in these analyses). We set alpha at .05, two-tailed, for all analyses.

To determine whether hormonal levels were continuously related to handedness index measures or whether effects were primarily due to differences in ex- treme-high or extreme-low subgroups, we next used between-groups analysis to examine differences in handedness between subjects assigned to low, middle, and high cortisol, testosterone, and ACTH groups. Subjects in the upper quartile of the sample were categorized in the high group, subjects in the second and third quartiles were categorized in the middle group, and subjects in the lower quartile were categorized in the low group. We conducted analysis of variance (ANOVA) and post-hoc tests where appropriate to determine the extent to which the two extreme groups differed from each other and from the middle group. We conducted this analysis on both HI and ABS-HI scores.

\section{RESULTS}

\section{Examination of Hand Preference Data}

Examination of handedness index (HI) data indicated that 18 animals (53\% of the subject sample) exhibited a positive HI score (indicative of right-hand bias) and 16 animals ( $47 \%$ of the subject sample) exhibited a negative HI score (indicative of left-hand bias). A singlesample $t$-test indicated that the mean HI score per subject (-.10) did not differ significantly from a null hypothesis distribution with a mean of 0 , indicating a lack of population-level bias in the direction of hand preference in adult rhesus macaques $(t(33)=1.40, p>.17)$. Regarding the relationship between hand preference direction and strength, we noted a significant negative correlation between $\mathrm{HI}$ and ABS-HI scores indicating that left-hand biased animals exhibited greater strength of preference than did right-hand biased animals (Pearson $r(32)=-.70, p<.0001)$.

\section{Relationship between Hand Preference and Cortisol}

Multiple regression analysis indicated a significant positive relation between plasma cortisol levels and $\mathrm{HI}$ scores $(t(3 / 31)=2.62 p \leqslant .02$; see Figure 1$)$ and a significant negative relation between plasma cortisol levels and ABS-HI scores $(t(3 / 33)=-2.31, p<.03)$. Linear analysis indicated a significant positive correlation between HI scores and plasma cortisol levels $(r(32)=.42$, $p<.02)$ and a near-significant negative correlation between ABS-HI scores and plasma cortisol levels $(r(32)=$ $-.32, p<.07)$. Analysis of extreme-score groups indicated a significant main effect of cortisol group on HI scores $(\mathrm{F}(2 / 31)=6.31, p \leqslant .005)$. Post hoc comparisons (Fischer's Protected Least Significant Difference test) revealed a significantly lower mean $\mathrm{HI}$ score for the low cortisol group (-.52) than for the middle and high cortisol groups (.02 and .03 , respectively), which did not differ significantly from each other $(p=.05)$. Further analysis indicated a group-level left-hand bias among animals in the low cortisol group (single-sample $t$-test, $\mathrm{t}(7)=$ 
$3.89, p<.006)$. Animals in the middle and high extreme groups did not show a significant group-level bias toward either the left or right hand (for the middle cortisol group, $t(17)=.19$; for the high cortisol group, $t(7)=$ .29). Extreme-groups analysis of data reflecting strength of handedness indicated no significant main effect of cortisol group on ABS-HI scores $(\mathrm{F}(2 / 31)=2.50)$.

\section{Relationship between Hand Preference and Testosterone}

Multiple regression analysis indicated a significant positive relation between plasma testosterone levels and HI scores $(t(3 / 31)=2.16, p \leqslant .04$; see Figure 2$)$ and a non-significant negative relation between plasma testosterone levels and ABS-HI scores $(t(3 / 31)=1.28)$. It must be noted that plasma testosterone levels were not significantly correlated with plasma cortisol levels $(r$ $(30)=.21, p>.25)$. Linear analysis indicated a significant positive correlation between HI scores and plasma testosterone levels $(r(30)=.39, p<.03)$ and a non-significant negative correlation between ABS-HI scores and plasma testosterone levels $(r(30)=-.24, p<.20)$. Analysis of extreme-score groups indicated a significant main effect of testosterone group on HI scores ( $\mathrm{F}$ $(2 / 29)=3.90, p \leqslant .04)$. Post hoc comparisons (Fischer's
Protected Least Significant Difference test) revealed a significantly lower mean HI score for the low testosterone group (-.34) than for the high testosterone group $(.20 ; p \leqslant .05)$. The mean HI score for the middle testosterone group (-.10) did not differ significantly from either the low or high testosterone groups. Further analysis indicated that animals in the low and middle testosterone groups did not show a significant grouplevel bias toward either the left or right hand (for the low testosterone group, $t(7)=2.11$; for the middle testosterone group, $t(15)=1.01)$; for the high cortisol group, $t(7)=.38$ ). Animals in the high cortisol group exhibited a group-level right-hand bias $(t(7)=2.79, p<$ $.03)$. Extreme-groups analysis of data reflecting strength of handedness indicated no significant main effect of testosterone group on ABS-HI scores $(\mathrm{F} 2 / 29)=1.20)$.

\section{Relationship between Hand Preference and ACTH}

Multiple regression analysis indicated no significant relation between levels of plasma ACTH sampled at adolescence and HI or ABS-HI scores sampled during adulthood $(t(3,31)=.35$ and 1.07 , respectively). Extreme-groups analysis indicated no significant main effect of testosterone group on HI or ABS-HI scores (F (2, $29)=.04$ and .20 , respectively).

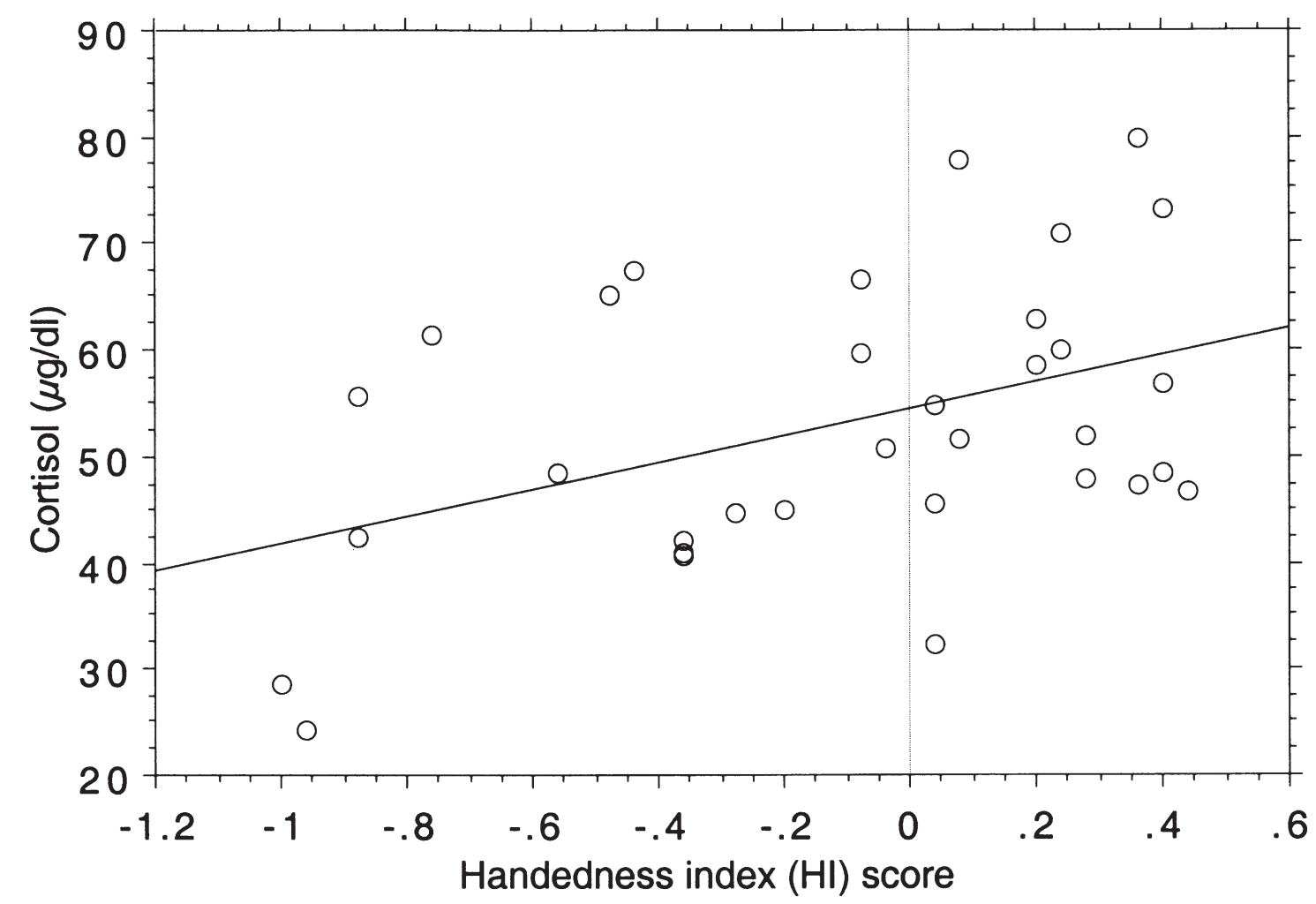

Figure 1. Scatterplot showing relationship between plasma cortisol levels and handedness index (HI) scores. A positive HI score is indicative of right-hand bias and a negative HI score is indicative of left-hand bias. 


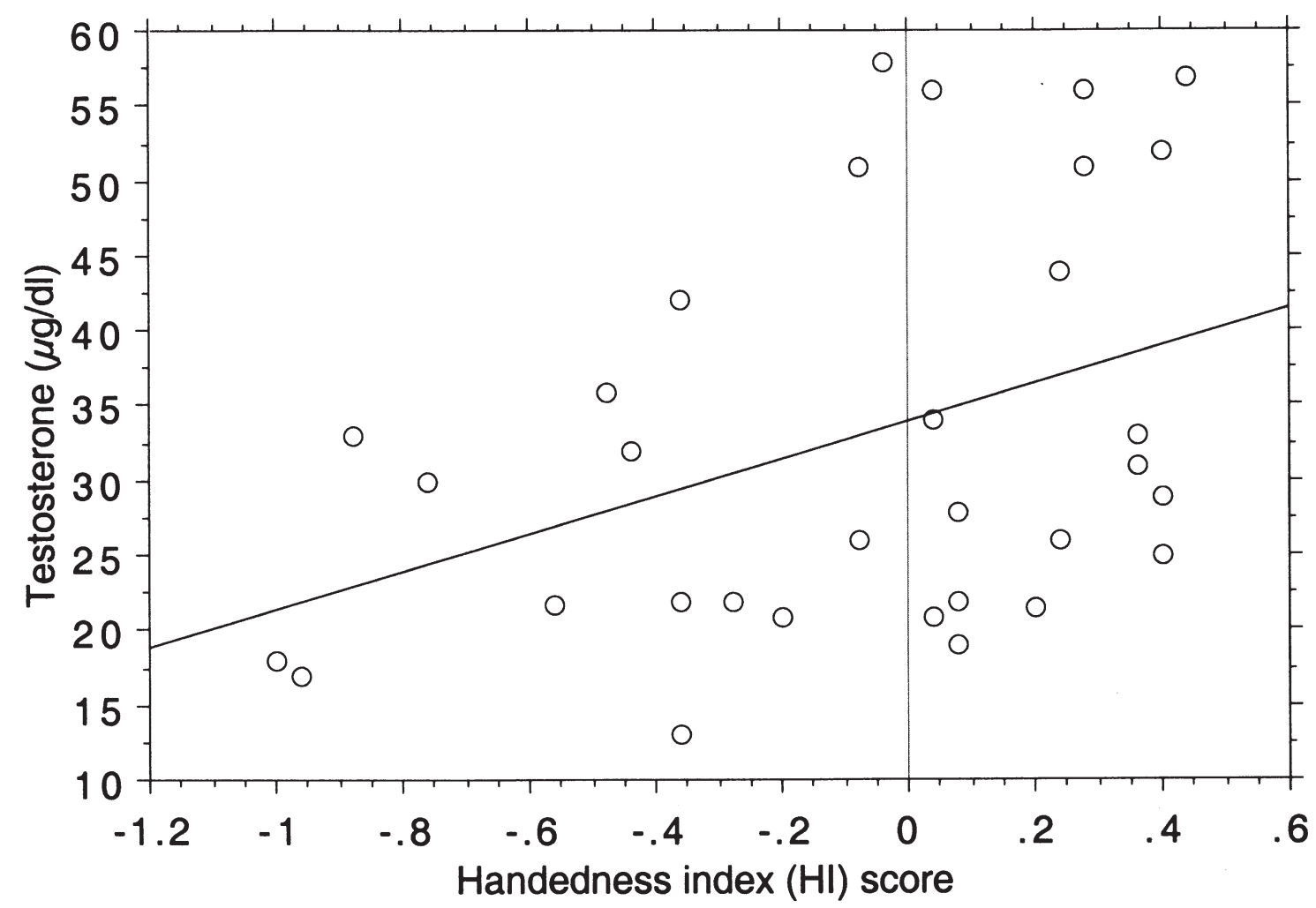

Figure 2. Scatterplot showing relationship between plasma testosterone levels and handedness index (HI) scores. A positive HI score is indicative of right-hand bias and a negative HI score is indicative of left-hand bias.

\section{DISCUSSION}

The results of this study demonstrate significant relationships between handedness and levels of plasma cortisol and testosterone in free-ranging rhesus macaques. Specifically, we noted significant positive correlations between levels of plasma cortisol and testosterone sampled during the juvenile and adolescent periods and lateral bias toward greater use of the right versus left hand sampled during adulthood. It must be noted that these findings do not confirm an etiological relationship among cortisol, testosterone, and the emergence of lateral bias. We cannot draw such a conclusion because we lack an early measure of lateral bias in our subjects. However, our data do provide evidence consistent with theories espousing a relationship between hormones and lateralization (see Geschwind and Galaburda 1985a, 1985b; Moffat and Hampson 1996) by indicating that hormonal functioning during adolescence is correlated with interindividual differences in the frequency of right- versus left-hand use in adulthood. It must also be noted that we found a significant negative correlation between cortisol and strength of lateral bias (independent of direction). This finding is of interest in view of research indicating that mixed handedness occurs at a higher rate in post-traumatic stress disorder patients than in experimental controls, suggesting that hemi- spheric lateralization is relevant to the pathophysiology of this disorder (Spivak et al. 1998).

When we conducted extreme-groups analysis on our cortisol data it became apparent that differences in the direction of hand preference were due primarily to the behavior of subjects in the low cortisol group as these animals were more biased toward use of their left hand than were animals in the middle or high cortisol groups. A different pattern emerged when we conducted extreme-groups analysis on our testosterone data as we noted a more continuous relationship between hemispheric specialization and levels of the male sex hormone. An important issue that remains to be resolved is whether interindividual differences in cortisol and testosterone levels are maintained across different stages of development even though absolute concentrations may vary considerably over time. In other words, can we accurately infer that extreme cortisol and testosterone concentrations during the juvenile and adolescent periods were produced by extreme hormonal concentrations in utero? The relevant studies to demonstrate this level of developmental continuity have yet to be undertaken, although it is known that levels of cortisol and testosterone remain stable over shorter periods of time and that there is a significant genetic contribution to hormonal production rates in adulthood, therefore making it reasonable to assume that interindividual differences in 
the concentrations of these hormones remain stable across different lifespan periods (Moffat and Hampson 1996).

One concern of this study is that we do not have a measure of hand preference prior to adolescence, which would allow us to more fully evaluate whether adolescent differences in hormone levels are causally related to adult hand preference or correlated with some third, and as yet, undefined variable. A second concern is whether the single measure of hand preference taken in this study reflects a stable trait in the subjects or whether the hand preference measure correlates with some aspect of competitive foraging (see Fagot et al. 1991) which in turn is associated with plasma hormone levels. At present we are addressing these concerns in a prospective study of free-ranging rhesus (using both males and females) in which we collect multiple measures of hand preference and plasma hormone levels beginning relatively early in life (at 2 years of age) and continuing into adulthood.

\section{ACKNOWLEDGMENTS}

The subjects for this research are part of a breeding colony supported by LABS of Virginia Inc. under Food and Drug Administration Contract 223-92-1101. LABS of Virginia Inc. is an AAALAC accredited institution. The LABS of Virginia Inc. Animal Care and Use Committee approved a research protocol for this study in accordance with and as required by the Animal Welfare Act. The authors thank Dr. Phillip Snoy (FDA) and LABS of Virginia Inc. for authorization to conduct this research.

\section{REFERENCES}

Bradshaw J, Rogers L (1993): The Evolution of Lateral Asymmetries, Language, Tool-Use, and Intellect. New York, Academic Press

Ciochon RL, Fleagle JG (1985): Primate Evolution and Human Origins. Menlo Park, CA, Benjamin/Cummings Publishing Company, Inc.

Drea CM, Wallen K, Akinbami MA, Mann DR (1995): Neonatal testosterone and handedness in yearling rhesus monkeys (Macaca mulatta). Physiol Behav 58:1257-1262

Fagot J, Drea CM, Wallen K (1991): Asymmetrical hand use in rhesus monkeys (Macaca mulatta) in tactually and visually regulated tasks. J Comp Psychol 105:260-268

Fleagle JG (1986): Primate Adaptations and Evolution. Orlando, FL, Academic Press, Inc.

Geschwind N, Galaburda AM (1985a): Cerebral lateralization: biological mechanisms, associations, and pathol- ogy: II. A hypothesis and a program for research. Arch Neurol 42:521-552

Geschwind N, Galaburda AM (1985b): Cerebral lateralization: biological mechanisms, associations, and pathology: III. A hypothesis and a program for research. Arch Neurol 42:634-654

Harris LJ, Carlson DF (1993): Hand preferences for visuallyguided reaching in human infants and adults. In Ward JP, Hopkins WD (eds), Primate Laterality: Current Behavioral Evidence of Primate Asymmetries. New York, Springer-Verlag, pp 285-306

Henry JP (1993): Psychological and physiological responses to stress: the right hemisphere and the hypothalamopituitary-adrenal axis, an inquiry into problems of human bonding. Integrative Physiolog Behav Sci 28:369-387

Kalin NH, Larson C, Shelton SE, Davidson RJ (1998): Asymmetric frontal brain activity, cortisol, and behavior associated with fearful temperament in rhesus monkeys. Behav Neurosci 112:286-292

Lindesay J (1987): Laterality shift in homosexual men. Neuropsychologia 25:965-969

MacNeilage PF, Studdert-Kennedy MG, Lindblom B (1987): Primate handedness reconsidered. Behav Brain Sci 10:247-303

McCormick CM, Witelson SF, Kingstone E (1990): Left-handedness in homosexual men and women: neuroendocrine implications. Psychoneuroendocrinology 15:69-76

Moffat SD, Hampson E (1996): Salivary testosterone levels in left- and right-handed adults. Neuropsychologia 34:225-233

Schneider ML, Suomi SJ (1992): Neurobehavioral assessment in rhesus monkey neonates (Macaca mulatta): developmental changes, behavioral stability, and early experience. Infant Behav Dev 15:155-177

Spivak B, Segal M, Mester R, Weizman A (1998): Lateral preference in post-traumatic stress disorder. Psychol Med 28:229-232

Warren JM (1980): Handedness and laterality in humans and other animals. Physiol Psychol 8:351-359

Westergaard GC, Champoux M, Suomi SJ (1997): Hand preference in infant rhesus macaques (Macaca mulatta). Child Dev 68:387-393

Westergaard GC, Kuhn HE, Suomi SJ (1998): Bipedal posture and hand preference in humans and other primates. J Comp Psychol 112:55-64

Westergaard GC, Lussier ID (1999): Left-handedness and longevity in primates. Int J Neurosci 99:79-87

Witelson SF (1991): Neural sexual mosaicism: sexual differentiation of the human temporo-parietal region for functional asymmetry. Psychoneuroendocrinology 16:131-153

Wittling W, Pfluger M (1990): Neuroendocrine hemisphere asymmetries: salivary cortisol secretion during lateralized viewing of emotion-related and neutral films. Brain Cognit 14:243-265 